\title{
Nutrition and its influence on periodontal disease
}

\author{
Shilpa Choudhari ${ }^{1, *}$, Vishakha Patil $^{2}$, Yogesh Khadtare ${ }^{3}$, Pallavi Patil ${ }^{4}$ \\ 1,3,4 Assistant Professor, ${ }^{2}$ Professor, Dept. of Periodontology, Bharati Vidyapeeth Deemed to be University Dental College, Pune,
} Maharashtra.

*Corresponding Author:

Email: shilpanalge9900@gmail.com

\begin{abstract}
Gingivitis and periodontitis are due to accumulation of plaque and calculus. Along with microorganisms there many underlying factors such as nutrition and general health of the patient is responsible for the periodontal disease progression. It is seen that there is direct relation between nutrition, immunity and susceptibility to the infection. Consistency of diet like consumption of fibrous food reduces plaque accumulation. Many studies proved that nutrition affects onset progression and healing of periodontal disease. It is very important to treat malnutrition when treating patients of periodontitis.
\end{abstract}

Keywords: Malnutrition, Periodontal disease.

\section{Introduction}

Comprehensive dental care requires dental professionals to assess the general health of their patients and to understand the implications of underlying factors that may impact oral health. One of these underlying factors are the interaction between nutritional status and the immune response to the bacterial challenge in periodontal disease. Alterations in immune response increase the risk and extent of infectious diseases such as periodontal disease. Since 1970s, the interrelationships of nutrition, immunity, and susceptibility to infection have received increasing attention and rigorous study.

It is generally acknowledged that Gingivitis and Periodontitis are result of an accumulation of supragingival and subgingival plaque or calculus or both. Even though the extent and intensity of the gingival inflammatory process are directly affected by the number and the virulence of dental plaque bacteria around the supragingival and subgingival margins of the teeth; it is indirectly affected systemically by the relative innate resistance of the periodontal tissues to infection. The dental health professional therefore has a responsibility not only to reduce the load of microorganisms by professional treatment and meticulous daily home plaque control programme, but also to help the patient increase the systemic resistance of the periodontal tissues by nutritional counseling. The latter process includes evaluating the diet that uses an adequate, well balanced food intake as a foundation (Glickman's clinical periodontology $7^{\text {th }}$ Edition W.B. Saunders (1990), ${ }^{1}$ company and Abraham L.N.(1996). ${ }^{2}$

Thus, inadequate nutrition may alter the host response to bacterial irritants and render the host more susceptible to establishment or progression of periodontal disease.

Nutritional disorders may arise due to:

1. Inadequate dietary intake.

2. Disturbances in the absorption of nutrients.
3. Economic and educational eliminations.

4. Self-imposed dietary restrictions.

5. Geographic isolation from adequate food supply.

The impact of nutrition on the health status can be best seen by examining extreme nutritional deprivation, which is seen to be less common in the developed countries. However numerous studies have conclusively demonstrated that the increased susceptibility to infection in a malnourished individual is due is a compromise in host defense factors.

The host defense factors that have an important bearing on periodontal health are:

a) Quantitative and qualitative characteristics of saliva and functional capacity of the salivary glands.

b) GCF production.

c) Repair.

d) Endocrine balance.

e) Integrity of the inflammatory - immune response.

f) Optimal function of the oral epithelium.

The exact mechanisms by which nutritional deficiencies modify periodontal destruction have not been precisely defined.

Alfano (1976) ${ }^{3}$ suggested that the following factors are affected in nutritional deficiencies:

a) Protein and urea contents of both saliva and crevicular fluid.

b) Integrity of the dentogingival barrier and the turnover of its constituent cells.

c) Mobilization and activation of PMNs in the early inflammatory response.

d) Activation of lymphocytes and the production of immunoglobulins in the immune response.

Extreme controversy has emanated from much of the research made into the role of nutrition in the etiology of periodontal diseases. 
According to Alfano(1976), ${ }^{3}$ these controversies are basically due to 6 problems encountered during research. They are:

1. The multifactorial etiology of periodontal disease makes the study of a specific predisposing factor very difficult or impossible.

2. Nutritional experiments on animals must be designed with both case control and pair -fed control groups.

3. Importance of using standardized periodontal indices and radiographic techniques must be recognized.

4. Animal model in experimental work should be chosen carefully and the animal should be susceptible to a deficiency of the nutrient under study.

5. Observations from nutritional studies must be assessed in relation to the length of the trial.

6. The statistical analysis of data from nutritional periodontitis studies is often complicated by factors such as number of control groups in animal experiments, death of animals as a direct result of nutritional upset, interactions between nutrients and the dominant and overriding effect of plaque on the recorded observations.

Unfortunately, substantial data have emanated from poorly controlled scientific studies on diet and nutrition and periodontal diseases where such information has not been analyzed vigorously by the appropriate statistical tests.

Diet and Nutrition: Nutrition is defined as the science of how the body utilizes food to meet the requirements for development, growth, repair and maintenance. ${ }^{4}$

Nutrition may influence the growth, development and metabolic activities of the periodontium. Tissues with a rapid rate of cell renewal such as periodontium depend on the ready availability of essential nutrients for the maintenance of their activities. They are therefore susceptible to the effect of malnutrition (Ramling swami1965, Winic and Noble 1966). ${ }^{5,6}$

Nutrition may influence pathogenesis of periodontal disease by affecting colonization and invasion of periodontal microbes, various parameters of host defense system and repair process that follows periods of active destruction (Enwonwu 1994). ${ }^{7}$

Studies have proved that the reason for lose teeth is poor diet. ${ }^{8,9}$ A persons diet can exert a topical or a systemic effect on the body and its tissues. Alteration in nutrition may have effect on tooth eruption, tooth development and in the maturation of dentin and enamel.

Periodontal lesion is essentially a wound, and sufficient host resources must be available for optimal healing to take place. According to Boyd (2003) ${ }^{10,11}$ nutrition affects immune system. Thus, it affects periodontal disease and its treatment.
It is important that dental professionals be able to identify patients at risk for poor nutrition, which may compromise their immune response and place them at higher risk for infection. Deterioration of oral health is highly correlated with deterioration of general health, making it essential that the patient be well nourished in order to respond to the challenge of infectious disease like periodontal diseases. ${ }^{12}$

Gingivitis and periodontitis are chronic infectious diseases. ${ }^{13}$ Gingivitis is defined as "inflammation of the gingiva in which the junctional epithelium remains attached to the tooth at its original level." ${ }^{14}$ In contrast, Periodontitis occurs when the "inflammatory process involves the gingiva and the periodontium resulting in loss of periodontal attachment." 14 The most recent National Health and Nutrition Examination Survey (NHANES III) found the prevalence of gingivitis in those aged 13 years and older, to be $54 \%$ and the prevalence of periodontitis (defined as attachment loss in at least one site) to be $53.1 \% .^{14}$

\section{Effect of Diet on Periodontal Disease a. Physical Consistency of Diet}

Numerous experiments in animals have shown that the physical character of diet may play some role in the accumulation of plaque and the development of gingivitis (Pelzer1940). ${ }^{16}$

Soft diet although nutritionally adequate lead to plaque and calculus formation (Burwasser and Hill $1939,{ }^{17}$ Lindhe and Wincen $1969^{18}$ and Pelzer $1940^{16}$ ). Hard and fibrous food provide surface cleansing action and stimulation of which result in less plaque and gingivitis, even if diet is nutritionally inadequate.

In humans, however studies have been unable to demonstrate reduced plaque formation when hard and fibrous foods are consumed (Krasse and Brill (1960). ${ }^{19}$ The discrepancy may be related to differences in tooth anatomy and to the fact that hard foods are fed to the experimental animals as the only diet whereas humans also have high sucrose content which favor the production of thick plaque (Carranza1996). ${ }^{20}$

In addition although it is frequently stated that fibrous food will provide gingival keratinization, the epithelium which lines the gingival crevice and is under constant challenge by subgingival plaque is not keratinized in the human. It has never been demonstrated that firm diet induces keratinization of this critically vulnerable epithelium in man.

Thus it is probable that the importance of dietary consistency on human plaque accumulation and gingival keratinization has been overemphasized (Alfano1976). ${ }^{3}$ Nevertheless a fibrous diet will minimize salivary gland atrophy and provide an increase in flow rates, total protein content and salivary amylase with potential benefits to oral health.

\section{b.Composition of Diet}


Although it is apparent that the composition of diet can alter the distribution and metabolism of microbes in the plaque, most of these changes occur in supragingival plaque which has ready access to dietary nutrients. The subgingival plaque is relatively shielded from direct dietary modification because of its location in gingival crevice. The composition of diet probably affects subgingival flora primarily by its systemic absorption and distribution in serum rather than by a direct effect in oral cavity.

It is proved that immunity depends on nutrition, ${ }^{21}$ because nutrients derived from food sources such as proteins, carbohydrates, and fats as well as micronutrients, vitamins, and minerals interact with immunie cells in the blood stream, lymph nodes and specialized immune system of gastrointestinal tract. ${ }^{22}$ Any type of infection has adverse effect on nutritional status $^{23}$ so infection, nutrition and immune system are interdependent on each other.

\section{Effect of Nutrition on Periodontal Disease ${ }^{24}$}

Many studies have proved that there is a possible mechanisms in which nutrition may influence periodontal disease onset, progression, and wound healing. For example, it has been suggested that in response to periodontal pathogens, the polymorphonuclear leukocytes (PMN) elaborate destructive oxidants, proteinases, and other factors. The balance between these factors, the antioxidants, and endogenously synthesized antiproteinases determine the extent of periodontal damage.

Malnutrition is characterized by marked tissue depletion of the key antioxidant nutrients, including gamma-glutamyl-cysteinyl-glycine (GSH), and impaired acute-phase protein response to infections. Acute-phase protein response plays a key role in promoting healing, and its deficit in malnutrition is due to impairment in the production and cellular action of the cytokines. Other features of malnutrition include inverted helper-suppressor $\mathrm{T}$ cell ratio, histaminemia, hormonal imbalance with increased blood and saliva levels of free cortisol, and defective mucosal integrity. Malnutrition has the potential to influence the prognosis of periodontal infections adversely. Additionally, malnutrition elicits adverse alterations in the oral microbial ecology as well as in the volume and the antibacterial and physicochemical properties of saliva.

\section{Summary}

Although the primary etiology of periodontal diseases is bacterial, host and environmental factors modulate the severity of disease. Host and environmental factors include genetics, chronic diseases such as osteoporosis and diabetes, tobacco use, socioeconomic status, educational level, frequency of dental visits, and nutrition. ${ }^{14}$

\section{References}

1. Glickman's clinical periodontology $7^{\text {th }}$ Edition W.B. Saunders (1990).

2. Abraham, S. (1996). "Characteristics of eating disorders among young ballet dancers." Psychopathology 29(4):223-229.

3. Alfano, M.C.: "Controversies, Perspectives and Clinical Implications of Nutrition in Periodontal Disease" Dent. Clin. North Amer. 20:519-548, 1976

4. Laura M. Romito, Introduction to nutrition and oral health Dent Clin N Am 47 (2003)187-207.

5. Chauhan S, Nayak NC, Ramalingaswami V: The heart and skeletal muscle in experimental protein malnutrition in Rhesus monkeys. Pathol 1965;90:301-309.

6. WINICK, M.; NOBLE, A. Cellular response in rats during malnutrition at various ages. J. Nutr., 89:300306,1966.

7. Enwonwu co. cellular and molecular effects of malnutrition and their, relevance to periodontal disease $\mathbf{J}$ Clin Periodontal 1994 21:643-657.

8. Marshall TA, Warren JJ, Hand JS, et al. Oral health, nutrient intake and dietary quality in the very old. J Am Dent Assoc 2002;133(10):1369-79.

9. Hung HC, Willett W, Ascherio A, et al. Tooth loss and dietary intake. J Am Dent Assoc 2003;134(9):1185-92.

10. Linda D, Boyd LD, Madden TE. Nutrition, infection, and periodonta disease. Dent Clin North Am 2003:47:337354.

11. Enwonwu CO, Phillips RS, Falkler WA Jr. Nutrition and oral infectious diseases: state of the science. Compend Contin Educ Dent 2002:23:431-434.

12. Hollister MC, Weintraub JA. The association of oral status with systemic health, quality of life, and economic productivity. J Dent Educ 1993;75:901-12.

13. Haake SK, Nisengard RJ, Newman MG, Miyasaki KT. Microbial interactions with the host in periodontal diseases. In: Newman MG, Takei HH, Carranza FA, editors. Carranza's clinical periodontology. $9^{\text {th }}$ edition. Philadelphia: W.B. Saunders; 2002. p.132-52.

14. Beck JD, Arbes SJ. Epidemiology of gingival and periodontal diseases. In: Newman MG, Takei HH, Carranza FA, editors. Carranza's clinical periodontology.9th edition. Philadelphia: W.B. Saunders; 2002. p. 74-94.

15. Robert e. Schifferle Periodontal disease and nutrition: separating the evidence from current fads. Periodontology 2000, Vol. 50, 2009, 78 - 89 .

16. Pelzer, R.: A study of the local oral effects of diet on the periodontal tissues and the gingival capillary structure. J. Amer. Dent. Assoc. 27:13;1940.

17. Burwasser P and Hill TJ, 1939, J Dent Res 18:389.

18. Lindhe J and Wicen P-O (1969) J Periodontol Ress 4:193.

19. Krasse B and Brill N (1960) Odontolrevy 11:152.

20. Carranza 1996.

21. Chandra RK. Nutrition and the immune system: an introduction. Am J ClinNutr 1997;66:440S-3S.

22. Cunningham-Rundles S. Analytical methods for evaluation of immune response in nutrient intervention. Nutr Rev 1998;56:S 27-37.

23. Scrimshaw NS, SanGiovanni JP. Synergism of nutrition, infection, and immunity: an overview. Am J ClinNutr 1997;66:464S-77S.

24. Neiva RF, Steigenga J, Al-Shammari KF, Wang H-L. Effects of specific Nutrients on periodontal disease onset, progression and treatment. J Clin Periodontol 2003;30:579-589. 